\title{
A case report with functional characterization of a HNF1B mutation (p.Leu168Pro) causing MODY5
}

\author{
Kei Yoshida ${ }^{1,2}$, Yuichi Mushimoto ${ }^{3}$, Kanako Tanase-Nakao ${ }^{1}$, Kazuhisa Akiba ${ }^{1}$, Kanako Ishii ${ }^{3}$, \\ Tatsuhiko Urakami ${ }^{2}$, Shigetaka Sugihara ${ }^{4}$, Toru Kikuchi ${ }^{5}$, Maki Fukami ${ }^{1}$, Satoshi Narumi ${ }^{1}$, \\ The Japanese Study Group of Insulin Therapy for Childhood and Adolescent Diabetes (JSGIT) \\ ${ }^{1}$ Department of Molecular Endocrinology, National Research Institute for Child Health and Development, Tokyo, \\ Japan \\ ${ }^{2}$ Department of Pediatrics and Child Health, Nihon University School of Medicine, Tokyo, Japan \\ ${ }^{3}$ Department of Pediatrics, Graduate School of Medical Sciences, Kyushu University, Fukuoka, Japan \\ ${ }^{4}$ Department of Pediatrics, Tokyo Women's Medical University Medical Center East, Tokyo, Japan \\ ${ }^{5}$ Department of Pediatrics, Saitama Medical University, Saitama, Japan
}

\begin{abstract}
We previously performed next-generation sequencing-based genetic screening in patients with autoantibodynegative type 1 diabetes, and identified the p.Leu168Pro mutation in HNF1B. Here, we report the clinical course of the patient and the results of functional characterization of this mutation. The proband had bilateral renal hypodysplasia and developed insulin-dependent diabetes during childhood. The pathogenicity of Leu168Pro-HNF1B was evaluated with three-dimensional structure modeling, Western blotting, immunofluorescence analysis and luciferase reporter assays using human embryonic kidney 293 cells. Three-dimensional structure modeling predicted that the Leu168 residue is buried in the DNA-binding Pit-Oct-Unc-specific $\left(\mathrm{POU}_{\mathrm{S}}\right)$ domain and forms a hydrophobic core. Western blotting showed that the protein expression level of Leu168Pro-HNF1B was lower than that of wild-type (WT) HNF1B. Immunofluorescence staining showed that both WT- and Leu168Pro-HNF1B were normally localized in the nucleus. The cells transfected with WT-HNF1B exhibited 5-fold higher luciferase reporter activity than cells transfected with an empty vector. The luciferase activities were comparable between WT-HNF1B/Leu168Pro-HNF1B and WT-HNF1B/empty vector co-transfection. In conclusion, Leu168Pro is a protein-destabilizing HNF1B mutation, and the destabilization is

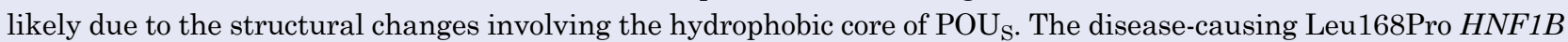
mutation is a loss-of-function mutation without a dominant-negative effect.
\end{abstract}

Key words: HNF1B, mutation, genetics, MODY5, multicystic dysplastic kidney

\section{Introduction}

Maturity-onset diabetes of the young (MODY) is a monogenic form of diabetes characterized by autosomal dominant inheritance, early onset and absence of B-cell autoimmunity. Fourteen different genes have been reported to be associated with MODY (1). MODY3 and MODY2 are the most common forms of MODY in Caucasians, accounting for $33-56 \%$ and $25-50 \%$ of cases, respectively (2-4). MODY5 is a relatively rare form of MODY, accounting for $2-6 \%$ of cases $(5,6)$. Genetic mutations in hepatocyte nuclear factor (HNF)-1B are known to cause MODY5 (7), which is characterized by various types of complications, the most common of which are renal malformations, such as horseshoe kidney, renal dysplasia, and multiple renal cysts (810). In addition, gonadal dysplasia, hepatic dysfunction, hyperuricemia, and bile duct dilatation have also been reported $(11,12)$. Patients with MODY5 have reduced insulin sensitivity to endogenous glucose production (13).

$H N F 1 B$ is located on chromosome $17 \mathrm{q} 12$ and is highly homologous to $H N F 1 A$. HNF1B is a tissuespecific transcription factor that forms homodimers or heterodimers with HNF1A and transactivates a variety of genes, such as albumin, a-fetoprotein, and glucose transporter $2(14,15)$. The crystal structure

Received: May 17, 2021 Accepted: July 20, 2021

Corresponding authorr: Satoshi Narumi, M.D., Ph.D., Department of Molecular Endocrinology, National Research Institute for Child Health and Development, 2-10-1 Okura, Setagaya-ku, Tokyo 157-8535, Japan

E-mail: narumi-s@ncchd.go.jp 
of the HNF1B DNA-binding domain revealed the presence of Pit-Oct-Unc $(\mathrm{POU})$-specific $\left(\mathrm{POU}_{\mathrm{S}}\right)$ and $\mathrm{POU}$ homeodomain $\left(\mathrm{POU}_{\mathrm{H}}\right)(16)$. According to the Human Protein Atlas database (https://www.proteinatlas.org/), HNF1B is expressed most abundantly in the kidney, and the second most abundantly in the exocrine pancreas. The HNF1B protein is also expressed in the genital tract, liver, gut, and lungs. This broad expression pattern of HNF1B is likely associated with multiple complications seen in the HNF1B defect (17). Various types of $H N F 1 B$ mutations have been reported, including 117 missense, 98 nonsense, 58 frameshift, and 24 splice site mutations, according to the Human Gene Mutation Database (http:// www.hgmd.cf.ac.uk/ac/).

We previously performed next-generation sequencing-based genetic screening in Japanese patients with autoantibody-negative type 1 diabetes (18) and identified the p.Leu168Pro mutation in $H N F 1 B$. However, the details of the examination and transition of treatment of the patient and functional characterization of the mutation were not reported. In this study, we report the clinical course of the patient and the results of the functional characterization of this previously uncharacterized mutation.

\section{Patient and Methods}

\section{Patient report}

The patient was a Japanese boy born at $36 \mathrm{wk}$ of gestational age and weighed 2,232 g (-1.2 SD) (Fig. 1). During pregnancy, no morphological abnormalities of the fetus, including renal malformations, were reported through routine ultrasonographic check-ups. Soon after birth, he was noted to have an imperforate anus. Ultrasonography revealed right renal hypodysplasia and left renal dysplasia. Blood examination showed that serum creatinine levels were increased, but no significant hyperglycemia was observed. Renograms demonstrated that the kidneys were nonfunctional.

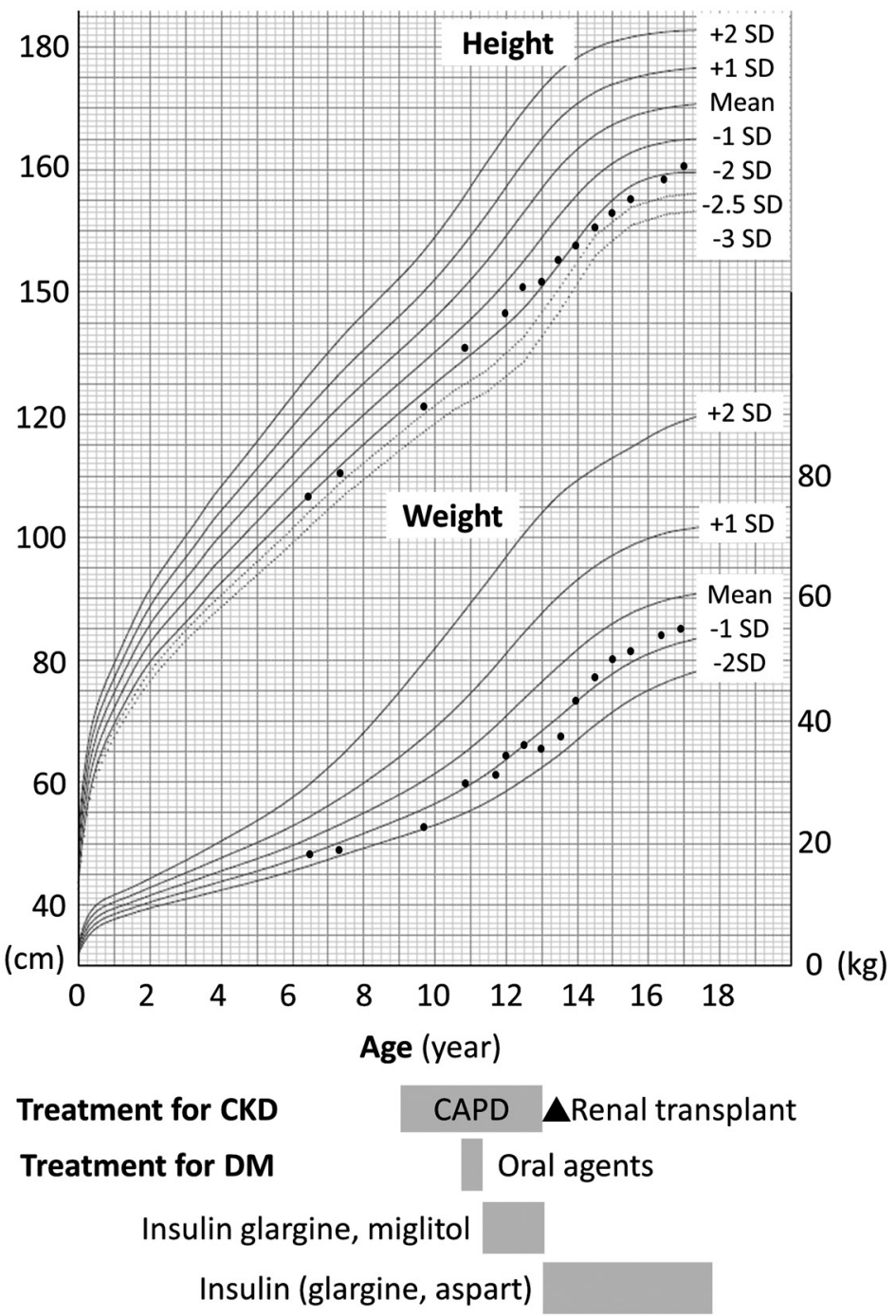

Fig. 1. Growth charts of the patient. The circles represent the patient's height and weight. Treatments for chronic kidney disease (CKD) and diabetes (DM) are aligned with ages of the growth charts. CAPD indicates continuous ambulatory peritoneal dialysis. 
He was diagnosed with chronic kidney disease (CKD) and was followed by pediatric nephrologists since then. Due to the progression of CKD, continuous ambulatory peritoneal dialysis was started at $9 \mathrm{yr}$ of age. At $10 \mathrm{yr}$ of age, a routine blood examination showed a high random plasma glucose level (19.6 mmol/mol; reference 3.9-11.1) and a high glycated hemoglobin level of $9.3 \%$ (78 mmol/ mol; reference 26-44), although he had no symptom of diabetes. Diabetic ketoacidosis was not observed. Both glutamic acid decarboxylase antibody and tyrosine phosphatase-like insulinoma antigen 2 antibodies were negative. He was initially treated with oral agents, including pioglitazone, miglitol, and glimepiride. Subsequently, insulin glargine was added to miglitol 4 mo after the diagnosis because his glycemic profiles worsened. Pioglitazone and miglitol were discontinued. He ultimately received a renal transplant at the age of $13 \mathrm{yr}$. Since the age of $13 \mathrm{yr}$, before the renal transplant, he was treated with a basal-bolus regimen with insulin aspart and insulin glargine. Based on this clinical course, the patient was diagnosed with autoantibody-negative type 1 diabetes (19). To clarify the molecular basis of autoantibody-negative type 1 diabetes, a genetic test was performed. After that, glycated hemoglobin levels were maintained at approximately $6 \%$. The random serum C-peptide level at the latest visit was $0.2 \mathrm{ng} / \mathrm{mL}$ (ref: > $0.6 \mathrm{ng} / \mathrm{mL}$ ). There was no family history of early onset diabetes or CKD.

\section{Ethics}

This study was approved by Ethics Committee of National Center for Child Health and Development and performed in accordance with the Declaration of Helsinki. Written informed consent for genetic and clinical investigations was obtained from the patients' parents.

\section{Three-dimensional structure modelling of the HNF1B protein}

The three-dimensional structure of HNF1B was modeled using data from the HNF1B-DNA complex (accession number 2H8R) obtained from the Protein Data Bank (https://www.rcsb.org/), and visualized using PyMOL version 0.99 (https://pymol.org/).

\section{Plasmids}

Human HNF1BcDNA was purchased from Addgene (Watertown, MA, USA; Plasmid \#31101). We created a vector expressing the N-terminal FLAG-enhanced green fluorescent protein-tagged-HNF1B fusion protein by inserting the FLAG sequence and HNF1B cDNA sequence into pEGFP-C1 using the Gibson assembly technique (NEBuilder HiFi DNA Assembly Master Mix; New England Biolabs, Ipswich, MA, USA). Leu168Pro was introduced using the standard site-directed mutagenesis technique.
We created a firefly luciferase reporter containing the promoter sequence of the human $A L B$ promoter gene corresponding to the $-163 /-1$ region. The human $A L B$ promoter sequence was amplified using the following primers: 5'- GGC AAG AAT ATT ATG AAT TTT GTA ATC G-3' (forward) and 5'- CAT TGT GCC AAA GGC GTG TGG GGT T -3' (reverse). The human ALB promoter sequence was cloned into pGL4.10 (Promega, Madison, WI, USA). To enhance HNF1-dependent transactivation, we chemically synthesized four tandem repeats of the HNF1 recognition sequence (5'- $\underline{\text { AGT CTA GTT AAT }}$ AAT CTA CCT AGT TAA TAA TCT ACG AAG TTA ATA ATC TAC GAA GTT AAT AAT CTA CAA TTA TTG GTT A -3'; HNF1B recognition sequence underlined), and inserted it into the native recognition site $(4 \times \mathrm{HNF}$-luc $)$.

\section{Cell culture and transient transfection}

Human embryonic kidney 293 cells were grown in DMEM supplemented with $50 \mathrm{IU} / \mathrm{mL}$ penicillin, $50 \mu \mathrm{g} /$ $\mathrm{mL}$ streptomycin, and $10 \%$ fetal bovine serum. Transient transfection of each effector plasmid with or without the luciferase reporter was performed using Lipofectamine 3000 (Thermo Fisher Scientific, Waltham, MA, USA) according to the manufacturer's protocol.

\section{Western blotting and immunofluorescence analysis}

For Western blotting, the cells were seeded in 24-well plates. At $48 \mathrm{~h}$ after transfection, the cells were harvested and subjected to sodium dodecyl sulphate-polyacrylamide gel electrophoresis (SDSPAGE). Western blotting was performed with a mouse monoclonal anti-FLAG M2 antibody (Sigma-Aldrich, St. Louis, MO, USA) at 500:1 dilution and anti-tubulin antibody (Abcam, Cambridge, UK; \#ab6160) at 1000:1 dilution. Horseradish peroxidase-conjugated goat antimouse IgG polyclonal antibody (Sigma-Aldrich) was used as the secondary antibody. Signals were detected using a chemiluminescence kit (Bio-Rad, Hercules, CA, USA).

For immunofluorescence analysis, the cells were seeded in 35-mm glass-bottom dishes. At 48 $\mathrm{h}$ after transfection, the cells were fixed with $4 \%$ paraformaldehyde in phosphate-buffered saline (PBS) at room temperature for $10 \mathrm{~min}$. After fixation, the cells were permeabilized with $0.1 \%$ Triton X-100 in PBS at room temperature for $10 \mathrm{~min}$ and incubated with $0.1 \%$ Hoechst 33342 in PBS for 10 min at room temperature. The cells were observed under an FV1000-D confocal microscope (Olympus, Tokyo, Japan).

\section{Luciferase reporter assays}

Human embryonic kidney 293 cells were seeded in 96-well plates (approximately 70-80\% confluence) and transiently transfected with $100 \mathrm{ng}$ of the $4 \times \mathrm{HNF}$ luc reporter vector together with $3 \mathrm{ng}$ of each $\mathrm{HNF} 1 \mathrm{~B}$ expression vector (WT or Leu168Pro) or an empty vector. 
Firefly luciferase activity was measured $48 \mathrm{~h}$ after transfection using ONE-Glo Luciferase Reporter Assay System (Promega) and a FlexStation 3 microplate reader (Molecular Devices, San Jose, CA, USA). Luciferase activity was measured in triplicate, and all assays were repeated at least thrice. Statistical significance was determined using the t-test. A two-tailed $P$-value with an alpha level for significance was set at $\leq 0.05$.

\section{Results}

\section{Detection of a novel HNF1B mutation p.Leu168Pro}

In our previous study (18), we performed a systematic investigation of genetic defects causing monogenic diabetes in Japanese patients clinically diagnosed with autoantibody-negative type 1 diabetes, and found that the patient described above had a heterozygous missense mutation of $H N F 1 B$ (c.503T >C, p.Leu168Pro) (18) (Fig. 2A). This mutation was not registered in any of the mutation/polymorphism databases, including
1000 Genomes Database (http://www.ncbi.nlm.nih.gov, gnomAD database (http://gnomad.broadinstitute.org), dbSNP database (http://www.ncbi.nlm.nih.gov/projects/ SNP), and Tohoku Medical Megabank database (https:// jmorp.megabank.tohoku.ac.jp). The amino acid leucine at position 168 of $\mathrm{HNF} 1 \mathrm{~B}$ and the corresponding residue of HNF1A is highly conserved across species (Fig. 2B). No other rare mutations were detected in the other tested diabetes-related genes. The parents declined familial genetic analysis.

\section{Location of Leu168 in the HNF1B protein}

The location of the Leu168 residue in the HNF1B protein was assessed using the crystal structure data of the HNF1B-DNA complex. Three-dimensional structure modeling of HNF1B showed that Leu168 is buried inside the $\mathrm{POU}_{\mathrm{S}}$ domain and likely configures a hydrophobic core (Fig. 2C).

(A)

\section{HNF1B protein}

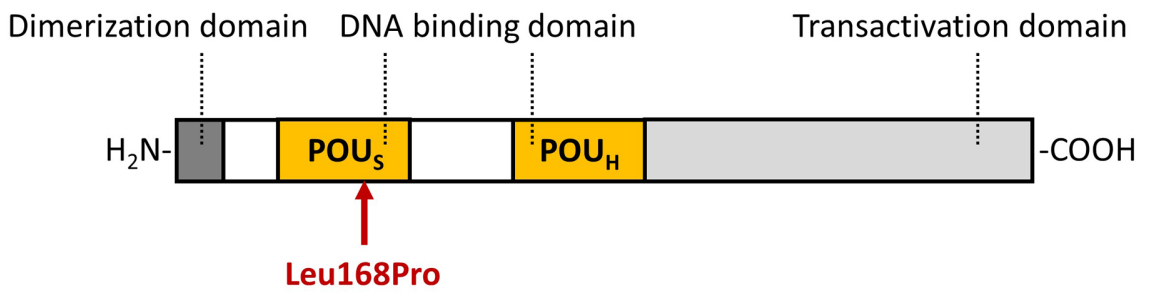

(B)

\begin{tabular}{|c|c|}
\hline Variant & $\mathbf{P}$ \\
\hline Human HNF1B & K T Q K R A A L Y T W Y V R K \\
\hline Mouse Hnf1b & $K T Q K R A A|L| Y T W Y \vee R K$ \\
\hline Chicken Hnf1b & $K T Q K R A A|L| Y T W Y \vee R K$ \\
\hline Xenopus Hnf1b & $K T Q K R A A|L| Y T W Y \vee R K$ \\
\hline Human HNF1A & $K T Q K R A A|L| Y T W Y \vee R K$ \\
\hline Mouse Hnf1a & $K T Q K R A A|L| Y T W Y \vee R K$ \\
\hline
\end{tabular}

(C)

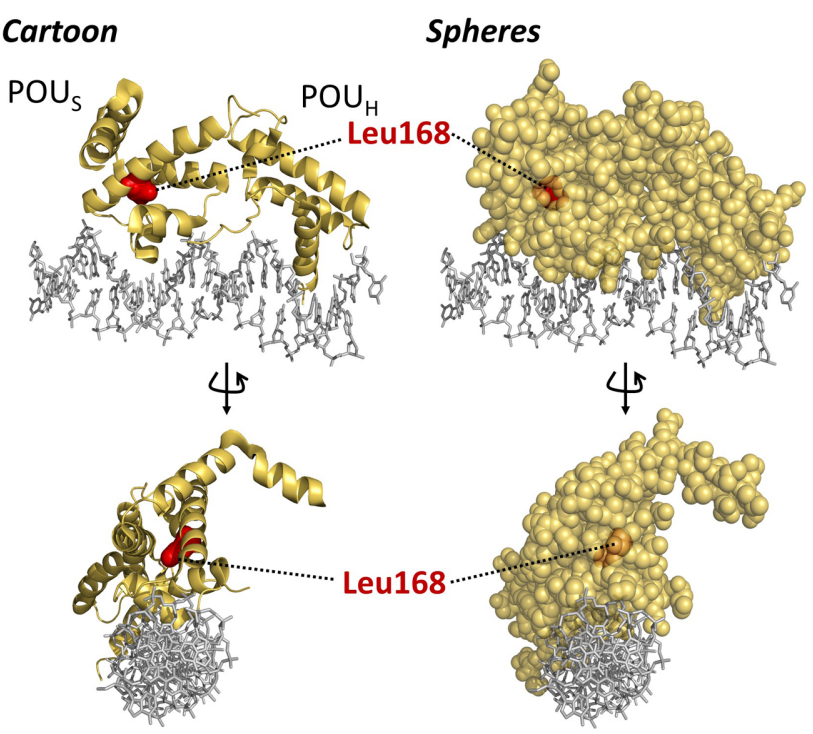

Fig. 2. The HNF1B mutation reported in this study. A: The structure of the HNF1B protein. Arrow indicates the position of the Leu168Pro mutation. DNA binding domains of HNF1B consist of the Pit-Oct-Unc (POU)-specific domain $\left(\mathrm{POU}_{\mathrm{S}}\right)$ and the POU homeodomain $\left(\mathrm{POU}_{\mathrm{H}}\right)$. B: Three-dimensional structure modeling of the DNA-binding domain $\left(\mathrm{POU}_{\mathrm{S}}\right.$ and $\left.\mathrm{POU}_{\mathrm{H}}\right)$ of the HNF1B protein. The Leu168 residue is colored in red. The residue is buried in the core of the $\mathrm{POU}_{\mathrm{S}}$ domain. C: Comparison of amino acid sequences of HNF1A and HNF1B among the species. The amino acid leucine at position 168 of the HNF1B and HNF1A proteins is highly conserved. 


\section{Protein expression and subcellular localization}

To verify the pathogenicity of the p.Leu168Pro $H N F 1 B$ mutation, we performed a series of expression experiments using human embryonic kidney 293 cells. Western blotting showed that the protein expression level of Leu168Pro-HNF1B was lower than that of WTHNF1B (Fig. 3A). Immunofluorescence staining showed that both WT- and Leu168Pro-HNF1B were normally localized in the nucleus (Fig. 3B).

\section{Luciferase reporter assays}

The cells transfected with WT-HNF1B exhibited 5 -fold higher luciferase activity of the $4 \times$ HNF-luc reporter than the cells transfected with the empty vector (Fig. 3C). This HNF1B-dependent transactivation was completely abolished in Leu168Pro-HNF1B. We then tested whether Leu168Pro-HNF1B interferes with the activity of WT-HNF1B (i.e., dominant negative effect) by co-transfection experiments. The luciferase activities were comparable between WT-HNF1B/Leu168ProHNF1B co-transfection and WT-HNF1B/empty vector co-transfection, indicating that Leu168Pro-HNF1B does not exert a dominant negative effect (Fig. 3D).

\section{Discussion}

We report the clinical course of an $H N F 1 B$ mutation-carrying Japanese boy with bilateral renal hypodysplasia and autoantibody-negative insulin-
(A)

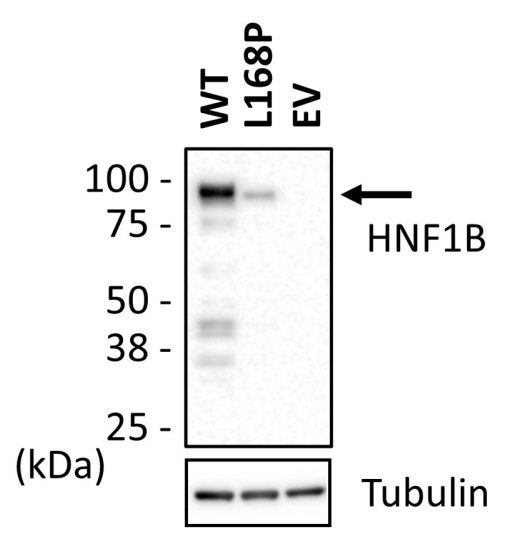

(C)

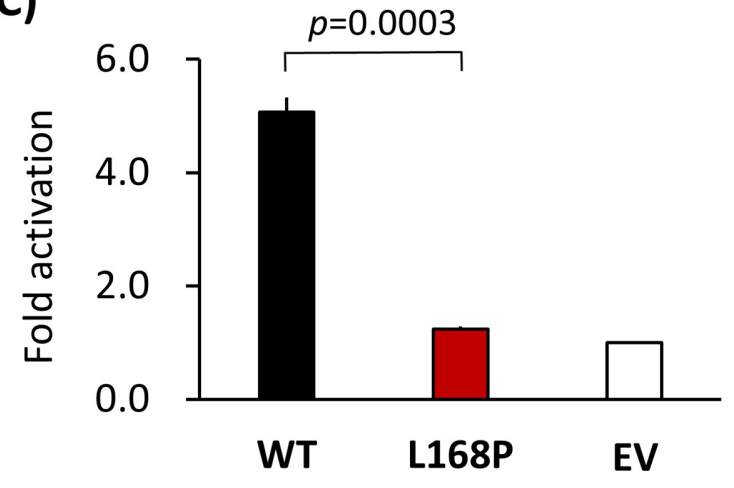

(B)

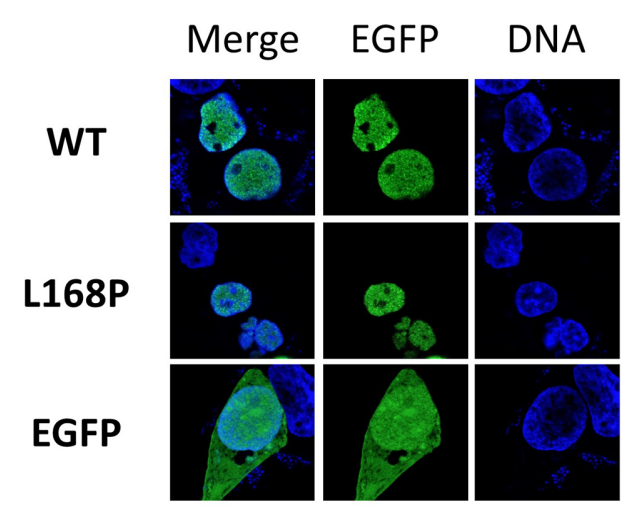

(D)

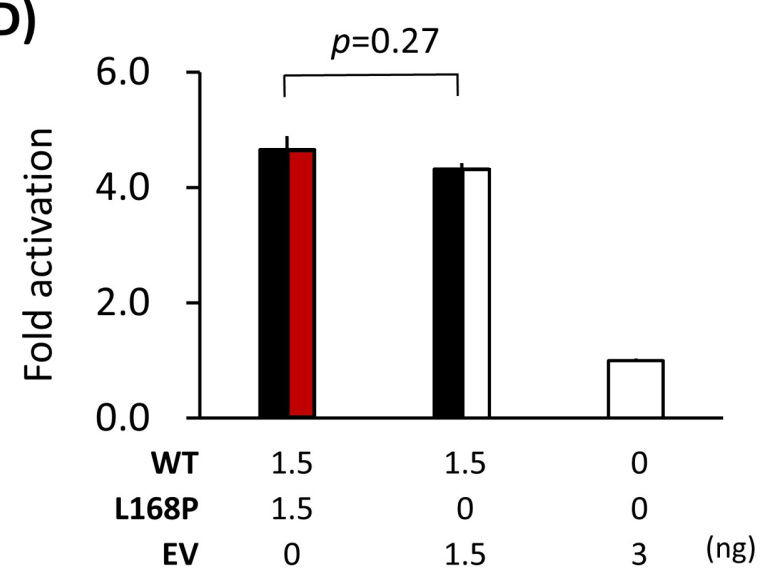

Fig. 3. Functional analysis of the Leu168Pro-HNF1B mutation. A: Protein expression levels of FLAG-EGFP-HNF1B [wildtype (WT) and Leu168Pro] were evaluated by Western blotting. The expected size of the FLAG-EGFP-HNF1B protein is indicated by an arrow. The expression level of the Leu168Pro protein was low. EV indicates empty vector. B: Intracellular localization of WT- and Leu168Pro-FLAG-EGFP-HNF1B. Hoechst 33342 (blue) was used for nuclear staining. C, D: Luciferase assays. Human embryonic kidney 293 cells were transfected with indicated amount (nanograms) of each HNF1B expression vector (WT and Leu168Pro). Left panel displays the results comparing WT-HNF1B (black bar) and Leu168Pro-HNF1B (red bar). Transactivating capacities of the FLAG-EGFP-HNF1B proteins (WT or Leu168Pro) were evaluated by the $4 \times$ HNF-luc reporter. Leu168Pro-HNF1B showed a negligible transacting capacity. Right panel displays the results comparing WT/Leu168Pro co-transfection (black and red bar) and WT/EV co-transfection (black and white bar). Dominant negative effect of the Leu168Pro mutation was assessed by performing the co-transfection experiment. The transactivation observed in WT/Leu168Pro co-transfection was comparable to that of WT/EV co-transfection. 
dependent diabetes in childhood. We characterized the Leu168Pro HNF1B mutation in vitro and confirmed that it was a loss-of-function mutation.

The HNF1B defect is the most commonly known monogenic form of CKD (17). Previous genetic studies of $H N F 1 B$ in CKD patients have reported that the proportion of mutation carriers was $5-31 \%$ (9, 20-26). Renal phenotypes of $H N F 1 B$ mutation carriers are variable, ranging from normal to renal dysplasia with end-stage renal failure (10). Therefore, it is difficult to predict which CKD patients have the HNF1B defect based on renal symptoms alone. Diabetes, the second most frequent phenotype of the HNF1B defect (27), has been observed in $5-50 \%$ of $H N F 1 B$ mutation carriers in previous studies (20-24). The presence of diabetes is a clinical key to suspect the HNF1B defect in CKD patients. Typically, onset of diabetes in the HNF1B defect is in the teens or twenties $(20,22,23,28)$, as in our patient. It is noteworthy that severity of diabetes is also variable: Dubois-Laforgue et al. reported that $49 \%$ of $140 H N F 1 B$ mutation-carrying patient with diabetes were treated with insulin at onset of diabetes, while $18 \%$ and $33 \%$ were treated with diet alone and oral hypoglycemic agents, respectively (28). Thus, even if the diabetes in a CKD patient is mild and can be controlled with oral medication alone, the HNF1B defect should be suspected. The previous studies reported that five out of six patients with the HNF1B defect treated with insulin immediately after diagnosis $(29,30)$. In our case, the patient was diagnosed with diabetes at $10 \mathrm{yr}$ of age but did not require insulin therapy for $3 \mathrm{yr}$. We speculate that the reason for the slow progression of our patient might be due to the fact that he had been receiving frequent blood tests for renal failure, resulting in very early detection of hyperglycemia.
We showed that Leu168Pro-HNF1B had an abrogated transactivating capacity on a reporter vector containing the four tandem repeats of the HNF1 recognition sequence. Co-transfection experiments of WT and $H N F 1 B$ mutations showed no dominant negative effects. Leu168Pro-HNF1B showed decreased protein expression. The most likely explanation for the low protein expression level is protein instability due to a structural change involving the $\mathrm{POU}_{\mathrm{S}}$ domain, as suggested by three-dimensional modeling, because the leucine to proline substitution would disorganize the hydrophobic core of $\mathrm{POU}_{\mathrm{S}}$.

\section{Conclusion}

We report the clinical course of the patient with the Leu168Pro HNF1B mutation and investigated the functional characteristics of the mutation. The results of our study indicate that (i) the substitution of Leu168 with proline probably affects the hydrophobic core of $\mathrm{POU}_{\mathrm{S}}$; (ii) the protein expression level of HNF1B is negatively affected by the Leu168Pro mutation; and (iii) Leu168Pro$H N F 1 B$ does not have a dominant negative effect. Our findings would contribute to a better understanding of the structure-function relationships of HNF1B.

Conflict of interests: The authors declare no conflicts of interest.

\section{Acknowledgements}

This study was supported by the National Center for Child Health and Development (2019A-1), the Manpei Suzuki Diabetes Foundation, and the Takeda Science Foundation.

\section{References}

1. Delvecchio M, Pastore C, Giordano P. Treatment options for MODY patients: A systematic review of jiterature. Diabetes Ther 2020;11: 1667-85. [Medline] [CrossRef]

2. Froguel P, Zouali H, Vionnet N, Velho G, Vaxillaire M, Sun F, et al. Familial hyperglycemia due to mutations in glucokinase. Definition of a subtype of diabetes mellitus. N Engl J Med 1993;328: 697-702. [Medline] [CrossRef]

3. Costa A, Bescós M, Velho G, Chêvre J, Vidal J, Sesmilo G, et al. Genetic and clinical characterisation of maturity-onset diabetes of the young in Spanish families. Eur J Endocrinol 2000;142: 380-6. [Medline] [CrossRef]

4. Lehto M, Wipemo C, Ivarsson SA, Lindgren C, Lipsanen-Nyman M, Weng J, et al. High frequency of mutations in MODY and mitochondrial genes in Scandinavian patients with familial early-onset diabetes. Diabetologia 1999;42: 1131-7. [Medline] [CrossRef]

5. Fendler W, Borowiec M, Baranowska-Jazwiecka A, Szadkowska A, Skala-Zamorowska E, Deja G, et al. Prevalence of monogenic diabetes amongst Polish children after a nationwide genetic screening campaign. Diabetologia 2012;55: 2631-5. [Medline] [CrossRef]

6. Kavvoura FK, Owen KR. Maturity onset diabetes of the young: clinical characteristics, diagnosis and management. Pediatr Endocrinol Rev 2012;10: 234-42. [Medline]

7. Horikawa Y, Iwasaki N, Hara M, Furuta H, Hinokio Y, Cockburn BN, et al. Mutation in hepatocyte nuclear factor-1 beta gene (TCF2) associated with MODY. Nat Genet 1997;17: 384-5. [Medline] [CrossRef]

8. Bohn S, Thomas H, Turan G, Ellard S, Bingham C, Hattersley AT, et al. Distinct molecular and morphogenetic properties of mutations in the human HNF1 beta gene that lead to defective kidney development. J Am Soc Nephrol 2003;14: $2033-41$. [Medline] [CrossRef]

9. Ulinski T, Lescure S, Beaufils S, Guigonis V, Decramer S, Morin D, et al. Renal phenotypes related to hepatocyte nuclear factor-1beta (TCF2) mutations in a pediatric cohort. J Am Soc Nephrol 2006;17: 497-503. [Medline] [CrossRef] 
10. Bingham C, Ellard S, Allen L, Bulman M, Shepherd M, Frayling T, et al. Abnormal nephron development associated with a frameshift mutation in the transcription factor hepatocyte nuclear factor-1 beta. Kidney Int 2000;57: 898-907. [Medline] [CrossRef]

11. Faguer S, Decramer S, Chassaing N, Bellanné-Chantelot C, Calvas P, Beaufils S, et al. Diagnosis, management, and prognosis of HNF1B nephropathy in adulthood. Kidney Int 2011;80: 768-76. [Medline] [CrossRef]

12. Bingham C, Ellard S, van't Hoff WG, Simmonds HA, Marinaki AM, Badman MK, et al. Atypical familial juvenile hyperuricemic nephropathy associated with a hepatocyte nuclear factor-1 beta gene mutation. Kidney Int 2003;63: 1645-51. [Medline] [CrossRef]

13. Brackenridge A, Pearson ER, Shojaee-Moradie F, Hattersley AT, Russell-Jones D, Umpleby AM. Contrasting insulin sensitivity of endogenous glucose production rate in subjects with hepatocyte nuclear factor-1 beta and -1alpha mutations. Diabetes 2006;55: 405-11. [Medline] [CrossRef]

14. Mendel DB, Hansen LP, Graves MK, Conley PB, Crabtree GR. HNF-1 alpha and HNF-1 beta (vHNF-1) share dimerization and homeo domains, but not activation domains, and form heterodimers in vitro. Genes Dev 1991;5: 1042-56. [Medline] [CrossRef]

15. Cereghini S. Liver-enriched transcription factors and hepatocyte differentiation. FASEB J 1996;10: 267-82. [Medline] [CrossRef]

16. Chi YI, Frantz JD, Oh BC, Hansen L, Dhe-Paganon S, Shoelson SE. Diabetes mutations delineate an atypical POU domain in HNF-1alpha. Mol Cell 2002;10: 1129-37. [Medline] [CrossRef]

17. Clissold RL, Hamilton AJ, Hattersley AT, Ellard S, Bingham C. HNF1B-associated renal and extra-renal disease-an expanding clinical spectrum. Nat Rev Nephrol 2015;11: 102-12. [Medline] [CrossRef]

18. Ushijima K, Fukami M, Ayabe T, Narumi S, Okuno M, Nakamura A, et al. Japanese Study Group of Insulin Therapy for Childhood and Adolescent Diabetes. Comprehensive screening for monogenic diabetes in 89 Japanese children with insulin-requiring antibody-negative type 1 diabetes. Pediatr Diabetes 2018;19: 243-50. [Medline] [CrossRef]

19. Mayer-Davis EJ, Kahkoska AR, Jefferies C, Dabelea D, Balde N, Gong CX, et al. ISPAD Clinical Practice Consensus Guidelines 2018: Definition, epidemiology, and classification of diabetes in children and adolescents. Pediatr Diabetes 2018;19(Suppl 27): 7-19. [Medline] [CrossRef]

20. Weber S, Moriniere V, Knüppel T, Charbit M, Dusek J, Ghiggeri GM, et al. Prevalence of mutations in renal developmental genes in children with renal hypodysplasia: results of the ESCAPE study. J Am Soc Nephrol 2006;17: 2864-70. [Medline] [CrossRef]

21. Decramer S, Parant O, Beaufils S, Clauin S, Guillou C, Kessler S, et al. Anomalies of the TCF2 gene are the main cause of fetal bilateral hyperechogenic kidneys. J Am Soc Nephrol 2007;18: 923-33. [Medline] [CrossRef]

22. Adalat S, Woolf AS, Johnstone KA, Wirsing A, Harries LW, Long DA, et al. HNF1B mutations associate with hypomagnesemia and renal magnesium wasting. J Am Soc Nephrol 2009;20: 1123-31. [Medline] [CrossRef]

23. Heidet L, Decramer S, Pawtowski A, Morinière V, Bandin F, Knebelmann B, et al. Spectrum of HNF1B mutations in a large cohort of patients who harbor renal diseases. Clin J Am Soc Nephrol 2010;5: 1079-90. [Medline] [CrossRef]

24. Madariaga L, Morinière V, Jeanpierre C, Bouvier R, Loget P, Martinovic J, et al. Severe prenatal renal anomalies associated with mutations in HNF1B or PAX2 genes. Clin J Am Soc Nephrol 2013;8: 1179-87. [Medline] [CrossRef]

25. Thomas R, Sanna-Cherchi S, Warady BA, Furth SL, Kaskel FJ, Gharavi AG. HNF1B and PAX2 mutations are a common cause of renal hypodysplasia in the CKiD cohort. Pediatr Nephrol 2011;26: 897-903. [Medline] [CrossRef]

26. Nakayama M, Nozu K, Goto Y, Kamei K, Ito S, Sato H, et al. HNF1B alterations associated with congenital anomalies of the kidney and urinary tract. Pediatr Nephrol 2010;25: 1073-9. [Medline] [CrossRef]

27. Bockenhauer D, Jaureguiberry G. HNF1B-associated clinical phenotypes: the kidney and beyond. Pediatr Nephrol 2016;31: 707-14. [Medline] [CrossRef]

28. Dubois-Laforgue D, Cornu E, Saint-Martin C, Coste J, Bellanné-Chantelot C, Timsit J, Monogenic Diabetes Study Group of the Société Francophone du Diabète. Diabetes, associated clinical spectrum, long-term prognosis, and genotype/phenotype correlations in 201 adult patients with hepatocyte nuclear factor 1B (HNF1B) molecular defects. Diabetes Care 2017;40: 1436-43. [Medline] [CrossRef]

29. Yorifuji T, Fujimaru R, Hosokawa Y, Tamagawa N, Shiozaki M, Aizu K, et al. Comprehensive molecular analysis of Japanese patients with pediatric-onset MODY-type diabetes mellitus. Pediatr Diabetes 2012;13: 26-32. [Medline] [CrossRef]

30. Horikawa Y, Enya M, Fushimi N, Fushimi Y, Takeda J. Screening of diabetes of youth for hepatocyte nuclear factor 1 mutations: clinical phenotype of HNF1 $\beta$-related maturity-onset diabetes of the young and HNF1 $\alpha$-related maturity-onset diabetes of the young in Japanese. Diabet Med 2014;31: 721-7. [Medline] [CrossRef] 